$\xi=\mathrm{\alpha}$

\title{
A systematic review of light therapy on mood scores in major depressive disorder: light specification, dose, timing and delivery
}

\author{
Mohammad A. Alotaibi, Mark Halaki, Chin-Moi Chow* \\ Exercise, Health \& Performance Faculty Research Group, Faculty of Health Sciences, \\ The University of Sydney, 75 East Street,Lidcombe, NSW 2141 \\ *Corresponding author E-mail: chin-moi.chow@sydney.edu.au
}

\begin{abstract}
Background: Depression is associated with prolonged disability, mortality, and morbidity. Ninety percent of patients with Major depressive disorder (MDD) have sleep problems. Light therapy has been shown to be effective in treating sleep disorders and MDD. This review aims to assess the characteristics (colour, intensity), exposure dose (duration and timing) and the mode of delivery (light boxes, visor etc) of light in reducing depression, measured by mood scores, in MDD.

Method: a systematic literature search was performed on 6 major databases. The Physiotherapy Evidence Database (PEDro) Scale was applied to assess study quality.

Result: Twenty-four articles satisfied the inclusion criteria. Effective light intensities varied from $176-10,000$ lux. Effective modes of delivery included light box, table lamp, visor and light emitting diode (LED) glasses. Effective light colours included white, green, and blue with the white colour being the most commonly used. The duration of light treatment ranged from $30 \mathrm{~min}$ to $2 \mathrm{~h}$, and evening light generally improved mood.

Conclusion: Light therapy, with exposure durations in the range of $30 \mathrm{~min}$ to $2 \mathrm{~h}$ per day, intensity range of 176 to 10,000 lux, in any of blue, green, or white light colour and exposure during morning mostly demonstrated a positive change in mood effects. Factors other than the light properties, such as anti-depressant medication use, depression episodes and severity, natural light exposure and sleep deprivation may confound the effects of light therapy.
\end{abstract}

Keywords: Bright Light; Depression; Natural Light; Non-Seasonal Affective Disorder; Phototherapy.

\section{Introduction}

Depression, described as an experience of low self-esteem, lack of interest, fatigue, suicidal thoughts, parasomnia and lack of appetite [1], is often associated with prolong disability, mortality, morbidity with numerous health issues and economic impact [2]. Major depressive disorder (MDD) is by far the most prevalent of all mood disorder diagnoses [3]. Depression, a topical issue, requires timely re-evaluation to report the degree of prevalence [4]. In 2005, depression was found to be the fourth most prevalent disorder managed by Australian general practitioners (GPs) [5]. In 2007, a survey conducted by the Australian Bureau of Statistics found that the prevalence of mental illness (including depression) in young adults was $26 \%$, which was one third higher than the overall adult population [6]. In 2010, depression remained the most prevalent disorder seen by the GPs [7]. Sleep disturbance is a key etiological factor of depression and has been found to be prevalent in $90 \%$ of the patients plagued with a major depression [8]. Troubled sleep and feelings of exhaustion and tiredness are commonly encountered during depression episodes [9-11]. Several sleep related issues faced by these patients are: not being able to sleep (initial insomnia), not sleeping as much as they normally do (mid insomnia), getting up several times at night and getting up earlier than normal in the morning (terminal insomnia), and finally not being able to go back to sleep once awake [12]. These obser- vations suggest sleep disturbances in circadian timing, and thus interventions to deal with the likely disturbances should consider the individual's circadian cycle [13].

Among methods used in treating sleep problems in depressive illness are psychotherapy or a combination of antidepressant medicines and psychotherapy [14]. However, these therapies do not completely target the link between depression and phase shifts in circadian sleep-wake cycles. Moreover, there are several sideeffects of antidepressant medications with greater rates of relapse [10]. Hence, sleep disturbances can be addressed through wake therapy (sleep deprivation) and light therapy [15]. Treatments for patients facing the most severe form of depression included a combination of wake therapy, light therapy and drug therapy [15]. The results were superior to that achieved by antidepressant drugs alone. The combined treatment showed an improvement of $27 \%$ in a single week, whereas, antidepressant medications reported an improvement of only $8-11 \%$ [15]. From among these therapies, light therapy offers several advantages, such as fast response and low degree of risk to patients [16]. Furthermore, combination of light treatment with drug and wake therapy have achieved faster results with higher success rates than antidepressants alone. Thus light therapy has been considered a better and more successful remedy for depressive disorders [17].

Treatment using light involves exposing patients to light at regular intervals for a certain time period. The devices used include light boxes that filter out ultraviolet waves, light emitting diodes (LED) 
technology that is smaller in size, fluorescent lamps that produce visible light (390-780 nm) and other devices to create bright light. Light therapy is particularly appropriate for patients who are resistant to and cannot tolerate medications. Given that the treatment is non-pharmacological and non-invasive, patients approve of it readily and at times, prefer it as well [18]. Light therapy has also been successfully used to treat seasonal affective disorder (SAD). $\mathrm{SAD}$ is a disorder that occurs when people experience depression intermittently in response to seasonal changes, most frequently during the fall and winter seasons due to short daylight. The photoperiod can be increased by five to six hours to reverse the seasonal changes using bright (2500 lux), full-spectrum light in the hours of morning, evening and at night [19]. Patients with Nonseasonal Affective disorder (NSAD) or MDD can also benefit from light treatments [20-24]. In fact bright-light therapy for MDD patients was $12-35 \%$ more beneficial than dim-light placebos or anti-depressants [25].

The effectiveness of light therapy for NSAD has been evaluated in three meta-analyses [26-28]. The reviews by [27], [28] concluded that light therapy was efficacious for NSAD, and bright light as an adjunct to antidepressant pharmacotherapy is effective for seasonal affective disorder and nonseasonal depression. The comprehensive analysis also suggests that light therapy can improve melatonin amplitude and cyclical sleep patterns along with improvement in depression score [29].

There are currently no guidelines to determine which patients will benefit from light therapy, as a few studies have demonstrated no impact of light therapy on patients with NSAD [20, 21, 30-32]. Furthermore, there has been no systematic evaluation and synthesis of the published literature regarding characteristics (colour intensity), exposure dose (duration and timing) and the mode of delivery (light boxes, visor etc) of light for use as a treatment for NSAD patients. The purpose of this review is to identify the elements that contributed to successful light therapy, as measured by the mood score, and identify any contravening factors and provide informed guidelines for future light therapy research for the treatment of depression.

\section{Method}

\subsection{Search strategy}

Database searches were performed in MEDLINE (via OvidSP, 1946 - April 10, 2015), PSYCHINFO (via OvidSP, 1806 - April 10, 2015), CINAHL (via Ebsco, 1982 - April 10, 2015), EMBASE (1980 - April 10, 2015), PubMed (1966 - April 10, 2015) and Cochrane Collaboration Library (1993 - April 10, 2015). The search terms included (depression or "major depressive disorder" or "non-seasonal affective disorder") and (light or "light therapy" or "bright light" or phototherapy). The search was limited to English language articles with subjects aged 18 and above. Articles that used light intervention on MDD were included. Articles that employed light intervention on seasonal affective disorder, on mental disorders (e.g., Alzheimer, dementia) other than MDD, on other diseases such as cancer, or on children, were excluded.

\subsection{Study selection/ quality assessments}

The Physiotherapy Evidence Database [33] for measuring study quality was used for the assessment of methodological quality in those articles.

\subsection{Data extraction}

The variables extracted were number of patients, sex, type of depression and diagnostic questionnaires used, mode of light delivery, light specifications (colour and intensity) for both intervention and placebo groups, timing and duration of treatment, mood outcomes from the light therapy and whether the participants were on antidepressant medications or not.

\section{Results}

The initial search identified 1662 articles after removal of duplicates. Following reviewing their titles, abstracts and/or full text, 24 articles were selected and further assessed using the PEDro scale. The excluded articles were either irrelevant to this review, or met the exclusion criteria. Five articles that examined the effect of sleep deprivation on depression as the main outcome were also excluded (Fig. 1)

\subsection{Demographic data and questionnaires used to assess mood}

Table 1 presents the extracted data listing demographic data, depression type, and the depression diagnostic tools employed by the respective studies. The total number of participants across the studies was 1024 (310 male, 626 female and 88 unreported sex). The age ranged from 35 to 70 years old. Diagnostic and Statistical Manual of Mental Disorders -III Revision and -IV (DSM-III-R and -IV) were prevalent diagnostic questionnaires in selected articles. Twenty-one studies used Hamilton Depression Rating Scale (HDRS) for scoring mood states [9], [11], [15], [16], [20-23], [30], [32], [34-44]. The other studies used scales such Geriatric Depression Scale, Major Depression Inventory and The International Classification of Diseases. Only a few studies identified patients' sleep related issues including hypersomnia and early or delayed sleep phases [10], [11], [30], [32].

\subsection{PEDro scale quality assessment}

Table 1 shows the results of quality assessment of the articles using the PEDro scale. According to the PEDro scale, the qualities of selected studies were generally high ranging from $7 / 11$ to $11 / 11$. 


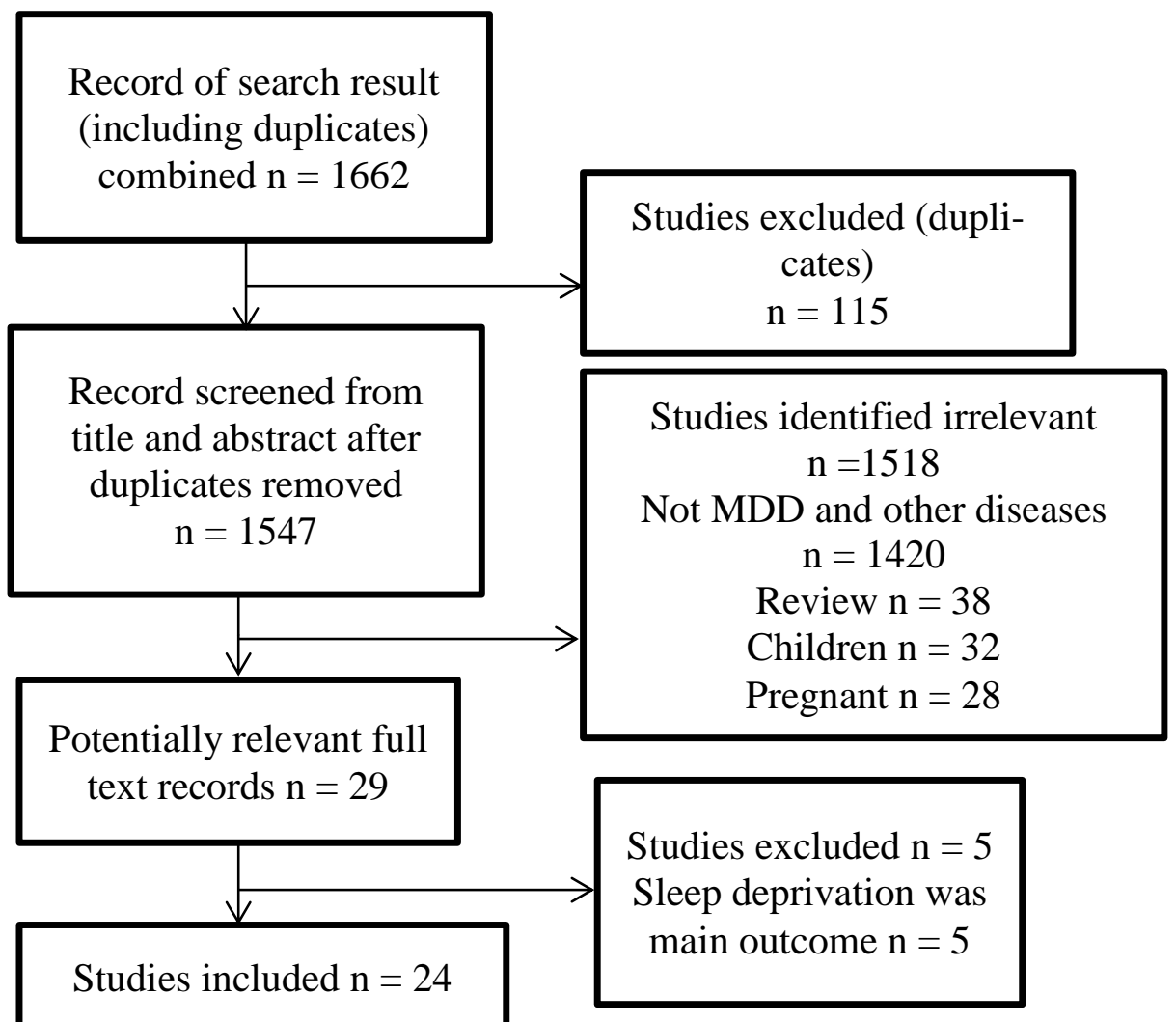

Fig. 1: Flow Chart of the Number of Articles Identified from the Search, Excluded and Included in the Review.

Table 1: Quality Assessment Results of Included Studies with Details of Subjects and Depression

\begin{tabular}{|c|c|c|c|c|c|}
\hline Author (year) & Sex & $\begin{array}{l}\text { Mean } \\
\pm \text { SD age }\end{array}$ & Type of depression & Diagnostic Questionnaire & PEDro Score Total \\
\hline Baumgartner et al.,[38] & $7 \mathrm{M} / 27 \mathrm{~F}$ & $\begin{array}{l}\text { BL: } 52.2 \pm 13.2 \\
\text { C: } 56.3 \pm 1.2\end{array}$ & MDD & DSM-III-R & 11 \\
\hline Beauchemin \& Hays[24] & $8 \mathrm{M} / 11 \mathrm{~F}$ & $39.0 \pm 11.5$ & \multicolumn{2}{|c|}{ MDD-single episode, MDD-recurrent, BD DSM-IV } & 7 \\
\hline Benedetti et al.,[41] & $6 \mathrm{M} / 24 \mathrm{~F}$ & $\begin{array}{l}\text { BL: } 53.0 \pm 10.0 \\
C: 56.2 \pm 12.3\end{array}$ & MDD, BD & DSM-IV & 10 \\
\hline Deltito et al.,[16] & $1 \mathrm{M} / 16 \mathrm{~F}$ & $36 \pm N R$ & MDD, BD, UBD & DSM-III-R & 10 \\
\hline Fritzsche et al.,[37] & $14 \mathrm{M} / 26 \mathrm{~F}$ & $\mathrm{~F} 47.0 \pm 11.0$ & MDD, SAD & DSM-IV & 10 \\
\hline Goel et al., [40] & $8 \mathrm{M} / 24 \mathrm{~F}$ & $43.1 \pm 12.0$ & MDD & DSM-IV & 9 \\
\hline Holsboer-Trachsler et al.,[42] & $22 \mathrm{M} / 22 \mathrm{~F}$ & $\mathrm{~F} 52.0 \pm 9.0$ & $\mathrm{MDD}, \mathrm{BD}, \mathrm{UBD}, \mathrm{DD}$ & DSM-III-R & 10 \\
\hline Kripke et al., [9] & $11 \mathrm{M} / 1 \mathrm{~F}$ & Mean age not reported & $\mathrm{MDD}, \mathrm{BD}$ & RDC & 9 \\
\hline Kripke et al., [22] & $50 \mathrm{M} / 1 \mathrm{~F}$ & $48.0 \pm N R$ & $\mathrm{MDD}, \mathrm{BD}$ & DSM-III & 9 \\
\hline Lieverse et al., [39] & $31 \mathrm{M} / 58 \mathrm{~F}$ & $\begin{array}{l}\text { BL: } 69.7 \pm 8.1 \\
\mathrm{C}: 69.0 \pm 6.1\end{array}$ & MDD & DSM-IV & 11 \\
\hline Loving et al., [15] & $2 \mathrm{M} / 11 \mathrm{~F}$ & $44.0 \pm N R$ & MDD & DSM-IV & 9 \\
\hline Loving et al., [30] & $34 \mathrm{M} / 47 \mathrm{~F}$ & $\mathrm{~F} 67.1 \pm 5.5$ & MDD & GDS, DSM-IV & 9 \\
\hline Mackert et al., [21] & $8 \mathrm{M} / 34 \mathrm{~F}$ & $54.0 \pm 13.0$ & MDD, BD & DSM-III-R,CGI & 11 \\
\hline Martiny et al., [44] & $32 \mathrm{M} / 70 \mathrm{~F}$ & $\begin{array}{l}\text { BL: } 43.0 \pm 15.1 \\
\mathrm{C}: 45.1 \pm 16.0\end{array}$ & MDD & \multicolumn{2}{|c|}{ MDI, PGWB, and SCL-90R 11} \\
\hline Martiny et al., [35] & $29 \mathrm{M} / 63 \mathrm{~F}$ & $\begin{array}{l}\text { BL: } 44.6 \pm 15.6 \\
\mathrm{C}: 46.3 \pm 15.2\end{array}$ & MDD & DSM-IV & 11 \\
\hline McEnany \& Lee [10] & $29 \mathrm{~F}$ & $\begin{array}{l}\text { Pre.M: } 37.5 \pm \mathrm{NR} \\
\text { Post.M: } 55.9 \pm \mathrm{NR}\end{array}$ & MDD & BDI, SCL-90R & 11 \\
\hline Prasko et al., [36] & $12 \mathrm{M} / 22 \mathrm{~F}$ & $\mathrm{~F} 42.1 \pm 10.0$ & MDD & DSM-III-R & 11 \\
\hline Rao et al., [31] & $53 *$ & $\begin{array}{l}\text { BL: } 53.1 \pm 1.0 \\
\text { C: } 52.1 \pm 1.0\end{array}$ & MDD & DSM-III-R, RDC and ICD9 & 11 \\
\hline Stewart et al., [43] & $7 \mathrm{M} / 23 \mathrm{~F}$ & $\begin{array}{l}\text { NSAD-AD: } 40.0 \pm 12.0 \\
\text { SAD: } 52.1 \pm 1.0\end{array}$ & NSAD-AD, SAD & DSM-III & 11 \\
\hline Strong et al., [34] & $9 \mathrm{M} / 26 \mathrm{~F}$ & $44.3 \pm 12.6$ & NSAD, SAD & DSM-IV,HAMD17,CGI & 11 \\
\hline Thalen et al., [11] & $12 \mathrm{M} / 51 \mathrm{~F}$ & $\begin{array}{l}\text { Female: } 49.1 \pm 1.0 \\
\text { Male: } 49.0 \pm 3.0\end{array}$ & MDD, SAD & DSM-III-R & 7 \\
\hline Volz et al., [20] & $5 \mathrm{M} / 25 \mathrm{~F}$ & $52.2 \pm 12.3$ & MDD & RDC, ICD-9 & 10 \\
\hline Yamada et al., [23] & $35^{*}$ & $47.6 \pm 2.2$ & MDD & DSM-III-R & 11 \\
\hline Yerevanian et al., [32] & $2 \mathrm{M} / 15 \mathrm{~F}$ & $41.5 \pm 13.5$ & MDD, SAD & DSM-III-R,BDI,GAS & 7 \\
\hline
\end{tabular}

BD: Bipolar depression; UBD: Unipolar depression; DD: Dysthymic Disorder; DSM: Diagnostic and Statistical Manual of Mental Disorders; GDS: Geriatric Depression Scale; HDRS: Hamilton Depression Rating Scale; ICD-9: The International Classification of Diseases; MDI: Major Depression Inventory; MDD: Major depressive disorder; NSAD: Non-seasonal affective disorder; PGWB: the Psychological General Well-Being Scale; RDC: Research Diagnostic Criteria; SAD: Seasonal affective disorder;
SCID: Structured Clinical Interview for DSM-IV Axis I Disorders; SCL90R: Symptom Check L; BL: Bright Light Group, C: Control Group; CGI: Clinical Global Impressions; Pre.M: Pre-Menopausal; Post.M: PostMenopausal; NSAD-AD: Non-seasonal Affective Disorder-Atypical Depression; HAMD-17: Hamilton Depression Rating Scale-17; GAS: Global Assessment Scale; NR: Not reported. *number in each sex not reported. 


\subsection{Mode of delivery and light specification (colour and intensity)}

Out of the 24 pertinent articles, only 5 reported a no effect while all other studies reported positive effects. Sixteen reported use of a light box, three employed a table lamp, one used a visor, three used a ceiling light, and one used a LED light for the light therapy (Table 2). Regardless of the mode of delivery, all modes showed positive mood effects.

Light sources of different colours showed positive effects on mood. Of the 24 studies, 21 used white light (16 had positive ef fects), two used blue light (both positive effects) and one green light (positive effect) (Table 2). Light intensities used were 176 lux for LED glasses, 1,000-3,000 lux for ceiling lights, 10,000 lux for visors, and 400-2,500 lux for table lamps and 2,500-10,000 lux for light boxes. Positive effects on mood were found across most intensities. Positive effects were observed using intensities ranging from 400 lux to 10,000 lux, although four of the five studies that found no mood effects used an intensity range between 2500 8,500 lux with one study indicating an intensity of $\geq 2,000$ lux. Some of the patients were already on anti-depressants.

Table 2 Effect of light source and its intensity, duration, and timing on mood changes, treatment duration and whether antidepressive medication was used during the trial. Light sources included light box, light box/lamp, visor, ceiling lamp and LED light.

\begin{tabular}{|c|c|c|c|c|c|c|c|c|c|c|c|}
\hline Study & $\begin{array}{l}\text { Mode of } \\
\text { Delivery }\end{array}$ & $\begin{array}{l}\text { Light } \\
\text { Colour }\end{array}$ & $\begin{array}{l}\text { Intensity } \\
\text { (lux) }\end{array}$ & $\begin{array}{l}\text { Placebo } \\
\text { group }\end{array}$ & Duration & Timing & $\begin{array}{l}\text { Treatment } \\
\text { Duration }\end{array}$ & $\begin{array}{l}\text { On Anti- } \\
\text { depressant } \\
\text { Medication }\end{array}$ & $\begin{array}{l}\text { Mood } \\
\text { effects }\end{array}$ & $\begin{array}{l}\text { HDRS Scores } \\
\text { Baseline }\end{array}$ & $\begin{array}{l}\text { Post- } \\
\text { treatment }\end{array}$ \\
\hline $\begin{array}{l}\text { Baumgartner et } \\
\text { al.,[38] }\end{array}$ & Light box & white & 2500 & red 50 lux & $2 \mathrm{~h}$ & a.m. & 1 week & No & Positive & $\begin{array}{l}\text { BL: } 19.2 \pm \\
4.3 \\
C: 19.9 \pm 4.0\end{array}$ & $\begin{array}{l}\text { BL: } 15.0 \pm \\
4.8 \\
C: 17.0 \pm \\
4.9\end{array}$ \\
\hline $\begin{array}{l}\text { Beauchemin \& } \\
\text { Hays[24] }\end{array}$ & Light box & white & 10000 & $\begin{array}{l}\text { white } 2500 \\
\text { lux }\end{array}$ & $30 \mathrm{~min}$ & a.m. & 6 days & Yes & Positive & NR & NR \\
\hline $\begin{array}{l}\text { Benedetti et } \\
\text { al.,[41] }\end{array}$ & $\begin{array}{l}\text { Table } \\
\text { lamp }\end{array}$ & green & 400 & other $^{b}$ & $30 \mathrm{~min}$ & a.m. & 4 weeks & Yes & Positive & $\begin{array}{l}\text { BL: } 23.7 \pm \\
6.9 \\
\text { C: } 22.6 \pm 4.9\end{array}$ & $\begin{array}{l}\text { BL:7.4 } \pm \\
07.7 \\
\text { C:13.1 } \pm \\
8.3\end{array}$ \\
\hline $\begin{array}{l}\text { Deltito et } \\
\text { al.,[16] }\end{array}$ & Light box & white* & 2500 & $\begin{array}{l}\text { white* } 400 \\
\text { lux }\end{array}$ & $2 \mathrm{~h}$ & a.m. & 1 week & No & Positive & $\begin{array}{l}\text { BL:31.0 } \pm \\
\text { NR } \\
\text { C:26.0 } \pm \\
\text { NR }\end{array}$ & $\begin{array}{l}\text { BL: } 6.0 \pm \\
\text { NR } \\
\text { C: } 19.0 \pm \\
\text { NR }\end{array}$ \\
\hline $\begin{array}{l}\text { Fritzsche et } \\
\text { al.,[37] }\end{array}$ & Light box & white & 2500 & red 50 lux & $2 \mathrm{~h}$ & a.m. & 2 week & Yes & Positive & $\begin{array}{l}\text { TSD-R } \\
\text { BL: } 17.1 \pm \\
6.0 \\
\text { C: } 15.0 \pm 3.0 \\
\text { TSD-NR } \\
\text { BL: } 16.1 \pm \\
4.0 \\
\text { C: } 15.0 \pm 4.1\end{array}$ & $\begin{array}{l}\text { TSD-R } \\
\text { BL:7.1 } \pm \\
7.0 \\
\text { C: } 6.1 \pm 2.1 \\
\text { TSD-NR } \\
\text { BL: } \\
11.1 \pm 3.1 \\
\text { C: } 12.0 \pm \\
5.1\end{array}$ \\
\hline Goel et al., [40] & Light box & white & 10,000 & other $^{b}$ & $1 \mathrm{~h}$ & a.m. & 5 weeks & No & Positive & $\begin{array}{l}\text { BL: } 15.1 \pm \\
3.0 \text { HDNAI: } \\
17.1 \pm 3.1 \\
\text { LDNAI: } \\
17.0 \pm 2.1\end{array}$ & $\begin{array}{l}\text { BL:7.1 } \pm \\
6.1 \\
\text { HDNAI: } \\
8.0 \pm 6.1 \\
\text { LDNAI: } \\
13.0 \pm 6.0\end{array}$ \\
\hline $\begin{array}{l}\text { Holsboer- } \\
\text { Trachsler et } \\
\text { al.,[42] }\end{array}$ & Light box & white & 5000 & $\begin{array}{l}\text { normal } \\
\text { treatment }\end{array}$ & $2 \mathrm{~h}$ & p.m. & 6 weeks & Yes & Positive & $\begin{array}{l}\text { BL:22.7 } \pm \\
5.3\end{array}$ & $\begin{array}{l}\text { BL:14.1 } \pm \\
5.8\end{array}$ \\
\hline $\begin{array}{l}\text { Kripke et al., } \\
\text { [9] }\end{array}$ & $\begin{array}{l}\text { Ceiling } \\
\text { light }\end{array}$ & white & $\begin{array}{l}1000- \\
2000\end{array}$ & red $25 \operatorname{lux}$ & $1 \mathrm{~h}$ & a.m. & 1 day & No & Positive & $\begin{array}{l}\text { BL: } 10.0 \pm \\
7.0 \\
\text { C: } 12.1 \pm 6.1\end{array}$ & NR \\
\hline $\begin{array}{l}\text { Kripke et al., } \\
\text { [22] }\end{array}$ & $\begin{array}{l}\text { Ceiling } \\
\text { light }\end{array}$ & white & $\begin{array}{l}2000- \\
3000\end{array}$ & red 50 lux & $2 \mathrm{~h}$ & p.m. & 1 week & No & Positive & $\begin{array}{l}\text { BL:100\% } \% \\
5 \% \\
\text { C:100\% } \\
5 \%\end{array}$ & $\begin{array}{l}\text { BL:86 \% } \pm \\
5 \% \\
\text { C:90\% } \% \\
5 \%\end{array}$ \\
\hline $\begin{array}{l}\text { Lieverse et al., } \\
\text { [39] }\end{array}$ & Light box & $\begin{array}{l}\text { pale } \\
\text { blue }\end{array}$ & 7500 & red 50 lux & $1 \mathrm{~h}$ & a.m. & 3 week & No & Positive & $\begin{array}{l}\text { BL: } 18.1 \pm \\
5.1 \\
\text { C:16.0 } \pm 4.1\end{array}$ & $\begin{array}{l}\text { BL: } 8.1 \pm \\
6.1 \\
C: 10.1 \pm \\
6.1\end{array}$ \\
\hline $\begin{array}{l}\text { Loving et al., } \\
\text { [15] }\end{array}$ & Light box & white & 10000 & red 100 lux & $30 \mathrm{~min}$ & a.m. & 1 week & Yes & Positive & $\begin{array}{l}\text { BL: } 24.0 \pm \\
9.8 \\
\text { C: } 18.8 \pm 7.2\end{array}$ & $\begin{array}{l}\text { BL: } 17.4 \pm \\
11.4 \\
C: 15.0 \pm \\
8.1\end{array}$ \\
\hline $\begin{array}{l}\text { Loving et al., } \\
\text { [30] }\end{array}$ & Light box & white & 8500 & red $<10$ lux & $1 \mathrm{~h}$ & $\begin{array}{l}\text { a.m./mid } \\
\text { /p.m. }\end{array}$ & 4 weeks & Yes & $\begin{array}{l}\text { No } \\
\text { effect }\end{array}$ & $\begin{array}{l}\text { BL: } 16.9 \pm \\
07.2 \\
\text { C: } 17.6 \pm \\
05.4\end{array}$ & $\begin{array}{l}\text { BL:11.1 } \\
07.1 \\
\text { C: } 11.3 \pm \\
06.0\end{array}$ \\
\hline $\begin{array}{l}\text { Mackert et al., } \\
\text { [21] }\end{array}$ & Light box & white & 2500 & red 50 lux & $2 \mathrm{~h}$ & a.m. & 1 week & No & $\begin{array}{l}\text { No } \\
\text { effect }\end{array}$ & $\begin{array}{l}\text { BL: } 19.1 \pm \\
4.0 \\
C: 19.0 \pm 4.0\end{array}$ & $\begin{array}{l}\text { BL: } 15.0 \pm \\
5.0 \\
\text { C: } 17.0 \pm \\
6.0\end{array}$ \\
\hline $\begin{array}{l}\text { Martiny et al., } \\
\text { [44] }\end{array}$ & Light box & white & 10000 & red 50 lux & $1 \mathrm{~h}$ & a.m. & 5 weeks & Yes & Positive & $\begin{array}{l}\text { BL: } 31.0 \pm \\
7.1 \\
\text { C: } 31.0 \pm 7.1\end{array}$ & $\begin{array}{l}\text { BL: } 12.08 \pm \\
8.08 \\
\text { C: } 15.03 \pm \\
9.0\end{array}$ \\
\hline $\begin{array}{l}\text { Martiny et al., } \\
\text { [35] }\end{array}$ & Light box & white & 10000 & red 100 lux & $1 \mathrm{~h}$ & a.m. & 5 weeks & Yes & Positive & $\begin{array}{l}\text { BL: } 10.0 \pm \\
5.1\end{array}$ & $\begin{array}{l}\text { BL: } 8.0 \pm \\
6.0\end{array}$ \\
\hline
\end{tabular}




\begin{tabular}{|c|c|c|c|c|c|c|c|c|c|c|c|}
\hline Study & $\begin{array}{l}\text { Mode of } \\
\text { Delivery }\end{array}$ & $\begin{array}{l}\text { Light } \\
\text { Colour }\end{array}$ & $\begin{array}{l}\text { Intensity } \\
\text { (lux) }\end{array}$ & $\begin{array}{l}\text { Placebo } \\
\text { group }\end{array}$ & Duration & Timing & $\begin{array}{l}\text { Treatment } \\
\text { Duration }\end{array}$ & $\begin{array}{l}\text { On Anti- } \\
\text { depressant } \\
\text { Medication }\end{array}$ & $\begin{array}{l}\text { Mood } \\
\text { effects }\end{array}$ & $\begin{array}{l}\text { HDRS Scores } \\
\text { Baseline }\end{array}$ & $\begin{array}{l}\text { Post- } \\
\text { treatment }\end{array}$ \\
\hline & & & & & & & & & & $\mathrm{C}: 12.0 \pm 5.0$ & $\begin{array}{l}\mathrm{C}: 08.1 \pm \\
5.0\end{array}$ \\
\hline $\begin{array}{l}\text { McEnany \& } \\
\text { Lee [10] }\end{array}$ & Visor & white & 10000 & $\begin{array}{l}\text { circadian } \\
\text { adaptation } \\
\text { glasses }\end{array}$ & $1 \mathrm{~h}$ & a.m. & 26 days & No & Positive & NR & NR \\
\hline $\begin{array}{l}\text { Prasko et al., } \\
\text { [36] }\end{array}$ & Light box & white & 5000 & red 500 lux & $2 \mathrm{~h}$ & a.m. & 3 weeks & Yes & Positive & $\begin{array}{l}\text { BL\&M: } \\
23.0 \pm 6.0 \\
\text { BL\&MP: } \\
23.0 \pm 3.1 \\
\text { DL\&M: } \\
24.1 \pm 3.1\end{array}$ & $\begin{array}{l}\text { BL\&M: } \\
17.0 \pm 11.0 \\
\text { BL\&MP: } \\
8.1 \pm 5.1 \\
\text { DL\&M: } \\
13.0 \pm 7.1\end{array}$ \\
\hline Rao et al., [31] & $\begin{array}{l}\text { Table } \\
\text { lamp }\end{array}$ & white & 2500 & red 50 lux & $2 \mathrm{~h}$ & a.m. & 1 week & No & $\begin{array}{l}\text { No } \\
\text { effect }\end{array}$ & NR & NR \\
\hline $\begin{array}{l}\text { Stewart et al., } \\
{[43]}\end{array}$ & Light box & white & 2500 & other* & $2 \mathrm{~h}$ & $\begin{array}{l}\text { a.m. \& } \\
\text { p.m. }\end{array}$ & 2 week & No & Positive & $\begin{array}{l}\text { NSAD-AD: } \\
16.0 \pm 03.1 \\
\text { SAD: } 16.0 \pm \\
4.1\end{array}$ & $\begin{array}{l}\text { NSAD- } \\
\text { AD: } \\
3.0 \pm 0.1 \\
\text { SAD:3.0 } \pm \\
0.1\end{array}$ \\
\hline $\begin{array}{l}\text { Strong et al., } \\
{[34]}\end{array}$ & $\begin{array}{l}\text { LED } \\
\text { glasses }\end{array}$ & $\begin{array}{l}\text { blue } \\
470 \mathrm{~nm}\end{array}$ & 176 & red 21 lux & $2 \mathrm{~h}$ & a.m. & 3 weeks & No & Positive & $\begin{array}{l}\mathrm{BL}: 20.0 \pm \\
3.1 \\
\mathrm{C}: 19.1 \pm 5.0\end{array}$ & $\begin{array}{l}\text { BL: } 10.0 \pm \\
5.0 \\
\text { C: } 6.0 \pm 3.1\end{array}$ \\
\hline $\begin{array}{l}\text { Thalen et al., } \\
\text { [11] }\end{array}$ & $\begin{array}{l}\text { Ceiling } \\
\text { light }\end{array}$ & white & 1500 & other $^{c}$ & $2 \mathrm{~h}$ & $\begin{array}{l}\text { a.m./ } \\
\text { p.m. }\end{array}$ & 10 days & Yes & Positive & $\begin{array}{l}\text { a.m.: } 15.6 \pm \\
1.3 \\
\text { p.m.:17.4 } \\
1.6\end{array}$ & $\begin{array}{l}\text { a.m.: } 13.0 \pm \\
1.7 \\
\text { p.m.: } 14.4 \pm \\
2.2\end{array}$ \\
\hline Volz et al., [20] & Light box & white & 2500 & $50 \operatorname{lux}$ & $2 \mathrm{~h}$ & a.m. & 1 week & No & $\begin{array}{l}\text { No } \\
\text { effect }\end{array}$ & $\begin{array}{l}\text { BL:20.0 } \\
\text { C: } 19.05\end{array}$ & $\begin{array}{l}\text { BL:15.0 } \\
\text { C: } 18.0\end{array}$ \\
\hline $\begin{array}{l}\text { Yamada et al., } \\
\text { [23] }\end{array}$ & Light box & white & 2500 & $\begin{array}{l}\text { yellow } 500 \\
\text { lux }\end{array}$ & $2 \mathrm{~h}$ & $\begin{array}{l}\text { a.m./ } \\
\text { p.m. }\end{array}$ & 1 week & No & Positive & $15.5 \pm 4.2$ & $9.6 \pm 5.3$ \\
\hline $\begin{array}{l}\text { Yerevanian et } \\
\text { al., [32] }\end{array}$ & $\begin{array}{l}\text { Table } \\
\text { lamp }\end{array}$ & white & $\geq 2000$ & other $^{c}$ & $2 \mathrm{~h}$ & $\begin{array}{l}\text { a.m./ } \\
\text { p.m. }\end{array}$ & 1 week & Yes & $\begin{array}{l}\text { No } \\
\text { effect }\end{array}$ & $23.0 \pm 9.0$ & $24.0 \pm 3.0$ \\
\hline
\end{tabular}

*Light colour not reported - white colour is assumed. Other: Studies compared to either medication ${ }^{\mathrm{a}}$, low or high density negative air ions ${ }^{\mathrm{b}}$, or Seasonal Affective disorder ${ }^{c}$. TSD-R: Total sleep deprivation responders. TSD-NR: Total sleep deprivation nonresponders. HDNAI: High density negative air ions LDNAI: Low density negative air ions; BL: Bright Light, C: Control, BL\&M: Bright light and medication; BL\&MP: Bright light and medication-like Placebo; DL\&M: Dim light and medication; NSAD-AD: Non Seasonal Affective Disorder-Atypical Depression; NR: Not Reported.

\subsection{Duration and timing of the light therapy}

The duration of light therapy employed ranged from $30 \mathrm{~min}$ to $2 \mathrm{~h}$ per day, and was generally effective on mood. However, four studies with $2 \mathrm{~h}$ light exposure and a study with $1 \mathrm{~h}$ light exposure showed no improvements on mood. The treatment period ranged from 1-4 weeks. Most of the studies $(n=17)$ delivered the treatment in the morning only, one study delivered the treatment in the morning and evening, three studies delivered the treatment in the morning or evening 2 studies delivered the treatment in the evening only and one study delivered the treatment in the morning or midday or in the evening. One of 1 study that delivered the therapy in the morning or midday or evening, 1 study of 3 that delivered the therapy in the morning or evening and 3 of 17 that delivered the therapy in the morning only failed to show efficacy.

\subsection{Taking anti-depressant medication during light therapy}

In 11 of the 24 studies, participants were on the anti-depressant medication during the light therapy. These studies showed positive effects of the therapy, except two studies $[30,32]$. Of the 13 studies where participants did not use anti-depressants, 10 showed positive effects.

\section{Discussion}

This paper assessed the properties of light, its timing, duration and intensity, and the mode of delivery that are therapeutic determinants in reducing depression in MDD, with a focus on mood state as the outcome measure. $79.2 \%(\mathrm{n}=19)$ of studies found that light therapy was effective in lifting mood state, consistent with the findings of previous reviews $[27,28]$. Despite the positive findings, it is challenging to clearly define the elements that contribute to the efficacy of light therapy. Factors other than the light proper- ties, such as anti-depressant medication use, depression episodes and severity, natural light exposure, treatment compliance and sleep deprivation were discussed to establish their impact on light therapy.

\subsection{Light characteristics}

The findings revealed that light intensities ranging from 176 to 10,000 lux showed positive effects on depression. However, previous studies demonstrated that an intensity of at least 2,000 lux was required to produce positive effects on depression [45], [46]. Remarkably, studies that used low intensity light $(176,400,1,000$ and 1,500 lux) also showed improvements in depression scores [9], [11], [34], [41] suggesting that even low intensity light was effective on mood alleviation.

All colours of light (blue, green and white) were effective in improving mood. White light is the most widely used source in $21 / 24$ studies. Its effectiveness may be due to the presence of blue and green colours as components of white light [47]. Indeed, the supra-chiasmatic nucleus ( $\mathrm{SCN}$ ) is sensitive to changes in bright light of white, blue and green and change of seasons [12], [23], [34], [39], [41]. The SCN controls circadian rhythms, which dictates the timing of physical, behavioural and mental patterns [9]. The output of SCN is modulated by light and darkness within an individual's surrounding on physiological rhythms [48]. Thus appropriate timing of light exposure can re-align altered sleepwake patterns [9].

Lewy et al. [13] suggested that evening light is more effective to correct sleep phase advance whereas morning lights has a therapeutic effect for phase delay. Unlike SAD, non-seasonal patients are more likely to have heterogeneous chronobiological patterns and could have either sleep phase advance or phase delay [28]. Of the 24 studies reviewed, one study demonstrated effective sleep phase delay by morning light timing and improved sleep length by aligning the timing of melatonin secretion [24] as well as enhancing mood [11], [24]. Given the scarce literature on light timing and mood, it is 
too soon to rule out the timing of light exposure (AM or PM) as insignificant in light treatment.

The optimal duration of light exposure needed for effective mood alleviation is also controversial. Wirz-Justice et al. [46] suggested that $2 \mathrm{~h}$ light exposure is more effective than $30 \mathrm{~min}$. However, the duration of light therapy that ranged from $30 \mathrm{~min}$ to $2 \mathrm{~h}$ showed positive effects on mood. Nevertheless, four studies that used $2 \mathrm{~h}$ light exposure failed to show efficacy. Importantly, if an exposure of as little as 30 min [15], [24], [41] can be demonstrated reproducibly to be efficacious for depression, other parameters defined, then a breakthrough in treatment can be claimed.

\subsection{Antidepressant medication - resistance to medica- tion}

Light therapy alone resulted in similar clinical effects as the use of anti-depressants alone [9], [10], [16], [22], [23], [34], [38-40], [43]. However, studies that combined antidepressant therapy with light therapy found greater improvement than with light therapy alone [11], [15], [24], [35-37], [41], [42], [44] suggestive of synergistic effects. On the other hand, the use of antidepressant medication with light therapy also showed no mood effects [30, 32] Such discrepancy in findings may be that the use of different study methodology made comparisons difficult. Discrepancy may also be explained by patients who may have demonstrated resistance to pharmacotherapy [49]. The interaction of the antidepressant effect of medication limits the understanding of the efficacy of light therapy. In addition, non-responders to therapies are common [50]. Therefore, it is necessary to identify patients groups who are homogenous, for example, those who are antidepressant medication-resistant prior to the commencement of light therapy, or patients either currently in or not currently in a depression episode.

\subsection{Depression episodes and severity}

Of the 24 studies, twenty-one studies indicated that the patients were studied during a depression episode. Patients continued to experience mild depression at the end of their treatment. This observation was despite the patients of these studies were on antidepressant medication [11], [15], [34], [36], [38], [44]. Lifestyle stressors and medical morbidity may influence mood. Of the 21 studies employing the HDRS scale, 5 studies showed no significant changes in the mood state following the light therapy [20], [21], [30-32]. Interestingly, these studies that showed no improvements reported high baseline HDRS scores (range 16.85 to 23.4, n=4). Whilst light exposure can lift mood, persistent stressors such as unresolved personal, psycho-social or economic factors, in those with high baseline scores may mask the effects of the light therapy. None of the 4 studies monitored these stressors.

\subsection{Natural light exposure}

Natural light and its daily exposure may have a direct impact on the mood state of patients undergoing light therapy. Indeed it represents a major confounding factor in these studies and could be the reason behind the results that showed minimal effects. Although two studies monitored the patients' natural light exposure using the Actillume (Ambulatory Monitoring INC., Ardsley, NY) [30] and an Actigraph (Philips Resprionics) [39] with a light sensor to monitor the sunlight exposure and adherence, neither studies reported the amount of light received nor discussed its confounding effects. Some authors advised patients to wear a dark cover that wrapped around the glasses if they were outdoor in order to not get much of the sunlight [10]. These findings suggest that, ideally, natural light exposure should be monitored during the treatment period, for example, with the Actiwatch2 (Philips Respironics) that is equipped with a light sensor, in future studies, would help unmask the true efficacy of light therapy.

\subsection{Treatment compliance}

Treatment compliance can directly impact on study outcomes. All 24 studies, including the 5 that did not show significant mood changes showed $100 \%$ study compliance with light exposure. At least in the current review study compliance does not appear to be a factor affecting the light therapy

\subsection{Sleep deprivation}

It has been well documented that sleep deprivation alleviates depression [51]. Loving et al. [15] found that patients with MDD who were treated with a combination of bright light, sleep deprivation, and medication improved $27 \%$ and a rapid response within 1 week. They noted that this response was superior when compared with the use of antidepressant drugs alone, which produced only a relative benefit of $8-11 \%$. Subsequently, Fritzsche et al. [37] showed that people who responded to sleep deprivation also responded to light therapy (regardless of intensity and colour) whereas, those who did not respond to sleep deprivation also did not respond to light therapy. This linked response is of interest. Should the finding prove to be reproducible, it would be feasible to conduct a sleep deprivation study to establish suitability for light therapy. It remains intriguing but unclear as to the mechanism that underlies the effectiveness of sleep deprivation and light therapy, and whether both treatments share a common path.

\section{Summary and conclusions}

Light therapy worked in 19/24 studies but there were no clear-cut mechanisms identified that would explain these results. Remarkably, light therapy, with light duration of a range of $30 \mathrm{~min}$ to $2 \mathrm{~h}$ per day, intensity ranging from 176 to 10,000 lux, colour (blue, green, or white), or its timing (AM or PM) mostly demonstrated a positive change in mood effects. However, it remains challenging to provide informed guidelines for light therapy as there may be numerous factors that could confound the study effects. The current data also do not permit determination of which patients will benefit from light therapy. Importantly, there is a need to understand the unique effect of light therapy without the imposing effect of antidepressants or natural light. There is also a need to establish that the paralleled mood enhancement effect seen with sleep deprivation and light therapy is a real phenomenon that can be reproduced. These foundation studies need to precede with welldesigned clinical trials of light and depression, which control for patients factors such as responsiveness to sleep deprivation, the severity of depression, life stressors, natural light exposure, depressant medications and drug resistance.

\section{Acknowledgements}

M. Alotaibi was awarded a PhD scholarship sponsored by Saudi Arabian Culture Mission (SACM), Canberra, Australia who represent the Ministry of Higher Education of the Saudi Arabian Government.

\section{References}

[1] L. Palagini, C. Baglioni, A. Ciapparelli, A. Gemignani, and D. Riemann, REM sleep dysregulation in depression: State of the art. Sleep Medicine Reviews $17 \quad$ (2013) 377-390. http://dx.doi.org/10.1016/j.smrv.2012.11.001.

[2] American, Psychiatric, and Association, Diagnostic and statistical manual of mental disorders: DSM-IV 1994: Washington DC.

[3] P. Waraich, E. Goldner , J. Somers, and L. Hsu Prevalence and incidence studies of mood disorders: a systematic review of the literature. Canadian Journal of Psychiatry 49 (2004) 124-138.

[4] Y. Conwell and D. Brent, Suicide and aging 1: patterns of psychiatric diagnoses. International Psychogeriatrics 7 (1995) 149-164. http://dx.doi.org/10.1017/S1041610295001943.

[5] H. Britt, G. Miller, S. Knox, et al. General practice activity in Australia 2004-05. General Practice Series No. 18 (2005); available online: 
http://www.aihw.gov.au/WorkArea/DownloadAsset.aspx?id=64424 56135 Accessed November 13, 2013

[6] Australian Bureau of Statistics. 4326.0 - National Survey of Mental Health and Wellbeing: Summary of Results, 2007 (2008); available online:

http://www.abs.gov.au/ausstats/abs@.nsf/Latestproducts/4326.0Mai n\%20Features32007?opendocument\&tabname=Summary\&prodno $=4326.0 \&$ issue $=2007 \&$ num $=\&$ view $=$ Accessed November 132013 .

[7] H. Britt, G. Miller, J. Charles, et al. General practice activity in Australia 2000-01 to 2009-10: 10 year data tables'. BEACH - Bettering the Evaluation and Care of Health (2010); available online: http://www.aihw.gov.au/WorkArea/DownloadAsset.aspx?id=64424 72732 Accessed November 15, 2014.

[8] S.R. Pandi-Perumal, A. Moscovitch, V. Srinivasan, et al., Bidirectional communication between sleep and circadian rhythms and its implications for depression: lessons from agomelatine. Progress in $\begin{array}{llll}\text { Neurobiology } & 88 & \text { (2009) } & \text { 264-271. }\end{array}$ http://dx.doi.org/10.1016/j.pneurobio.2009.04.007.

[9] D.F. Kripke, S.C. Risch, and D. Janowsky, Bright white light alleviates depression. Psychiatry Research 10 (1983) 105-12. http://dx.doi.org/10.1016/0165-1781(83)90109-9.

[10] G.W. McEnany and K.A. Lee, Effects of light therapy on sleep, mood, and temperature in women with nonseasonal major depression. Issues in Mental Health Nursing 26 (2005) 781-94. http://dx.doi.org/10.1080/01612840591008410.

[11] B.E. Thalen, B.F. Kjellman, L. Morkrid, and L. Wetterberg, Melatonin in light treatment of patients with seasonal and nonseasonal depression. Acta Psychiatrica Scandinavica 92 (1995) 274-84. http://dx.doi.org/10.1111/j.1600-0447.1995.tb09583.x.

[12] M. Berk, Sleep and depression Theory and practice. Australian Family Physician 38 (2009) 302-304.

[13] A.J. Lewy, Sack R. L., \& Singer, C. M., Treating phase typed chronobiologic sleep and mood disorders using appropriately timed bright artificial light. Psychopharmacology Bulletin 21 (1985) 368372 .

[14] G. Glickman and J. Hanifin, inferior retinal light exposure is more effective than superior retinal exposure in suppressing melatonin in humans. Journal of Biological Rhythms 18 (2003) 71-79. http://dx.doi.org/10.1177/0748730402239678.

[15] R.T. Loving, D.F. Kripke, and S.R. Shuchter, Bright light augments antidepressant effects of medication and wake therapy. Depression and Anxiety 16 (2002) 1-3. http://dx.doi.org/10.1002/da.10036

[16] J.A. Deltito, M. Moline, C. Pollak, L.Y. Martin, and I. Maremmani, Effects of phototherapy on non-seasonal unipolar and bipolar depressive spectrum disorders. Journal of Affective Disorders 23 (1991) 231-237 available online.

[17] R.T. Joffe, D.E. Moul, R.W. Lam, et al., Light visor treatment for seasonal affective disorder: A multicenter study. Psychiatry Research 46 (1993) 29-39 available online.

[18] R. Lieverse, E.J. Van Someren, M.M. Nielen, et al., Bright light treatment in elderly patients with nonseasonal major depressive disorder: a randomized placebo-controlled trial. Archives of Gen$\begin{array}{lllll}\text { eral } & \text { Psychiatry } & 68 & \text { (2011) }\end{array}$ http://dx.doi.org/10.1001/archgenpsychiatry.2010.183.

[19] M. Terman, J.S. Terman, F.M. Quitkin, et al., Light therapy for seasonal affective disorder: A review of efficacy. Neuropsychopharmacology 2 (1989) 1-22 available online.

[20] H.P. Volz, A. Mackert, R.D. Stieglitz, and B. Muller-Oerlinghausen, Effect of bright white light therapy on non-seasonal depressive disorder. Preliminary results. Journal of Affective Disorders 19 (1990) 15-21. http://dx.doi.org/10.1016/0165-0327(90)90004-R.

[21] A. Mackert, H.P. Volz, R.D. Stieglitz, and B. Muller-Oerlinghausen, Phototherapy in nonseasonal depression. Biological Psychiatry 30 (1991) 257-68. http://dx.doi.org/10.1016/0006-3223(91)90110-8.

[22] D.F. Kripke, D.J. Mullaney, M.R. Klauber, S.C. Risch, and J.C Gillin, Controlled trial of bright light for nonseasonal major depressive disorders. Biological Psychiatry 31 (1992) 119-34. http://dx.doi.org/10.1016/0006-3223(92)90199-A.

[23] N. Yamada, M.T. Martin-Iverson, K. Daimon, T. Tsujimoto, and S. Takahashi, Clinical and chronobiological effects of light therapy on nonseasonal affective disorders. Biological Psychiatry 37 (1995) 866-73. http://dx.doi.org/10.1016/0006-3223(94)00221-N.

[24] K. Beauchemin and P. Hays, Phototherapy is a useful adjunct in the treatment of depressed in-patients. Acta Psychiatrica Scandinavica 95 (1997) 424-427 available online: http://dx.doi.org/10.1111/j.1600-0447.1997.tb09656.x.

[25] D.F. Kripke, Light treatment for nonseasonal depression: speed, efficacy, and combined treatment. Journal of Affective Disorders 49 (1998) 109-117. http://dx.doi.org/10.1016/S0165$\underline{0327(98) 00005-6}$
[26] A. Tuunainen, D.F. Kripke, and T. Endo, Light therapy for nonseasonal depression. Cochrane Database of Systematic Reviews (2004). http://dx.doi.org/10.1002/14651858.CD004050.pub2.

[27] R.N. Golden, B.N. Gaynes, R. Ekstrom, et al., the Efficacy of Light Therapy in the Treatment of Mood Disorders: A Review and MetaAnalysis of the Evidence. The American Journal of Psychiatry 162 (2005) 656-662 available online: http://dx.doi.org/10.1176/appi.ajp.162.4.656.

[28] C. Even, C.M. Schroder, S. Friedman, and F. Rouillon Efficacy of light therapy in nonseasonal depression: A systematic review. Journal of Affective Disorders 108 (2008) 11-23. http://dx.doi.org/10.1016/j.jad.2007.09.008.

[29] A.M. Kledzik and M.C. Thorne, The role of melatonin in psychiatric disorders. 2011. 49-55.

[30] R.T. Loving, D.F. Kripke, J.A. Elliott, N.C. Knickerbocker, and M.A. Grandner, Bright light treatment of depression for older adults [ISRCTN55452501]. BMC Psychiatry 5 (2005) 41 http://dx.doi.org/10.1186/1471-244X-5-41.

[31] M.L. Rao, B. Muller-Oerlinghausen, A. Mackert, et al., Blood serotonin, serum melatonin and light therapy in healthy subjects and in patients with nonseasonal depression. Acta Psychiatrica Scandinavica 86 (1992) 127-32. http://dx.doi.org/10.1111/j.16000447.1992.tb03240.x.

[32] B.I. Yerevanian, J.L. Anderson, L.J. Grota, and M. Bray, Effects of Bright Incandescent Light on Seasonal and Nonseasonal Major Depressive Disorder. Psychiatry Research 18 (1986) 355-364 http://dx.doi.org/10.1016/0165-1781(86)90020-X

[33] The Physiotherapy Evidence Database. PEDro scale. 21 June 1999; available online: http://www.pedro.org.au/english/downloads/pedro-scale/ Accessed January 102015.

[34] R.E. Strong, B.K. Marchant, F.W. Reimherr, et al., Narrow-band blue-light treatment of seasonal affective disorder in adults and the influence of additional nonseasonal symptoms. Depression and Anxiety 26 (2009) 273-8. http://dx.doi.org/10.1002/da.20538.

[35] K. Martiny, M. Lunde, M. Unden, H. Dam, and P. Bech, The lack of sustained effect of bright light, after discontinuation, in nonseasonal major depression.[Erratum appears in Psychol Med. 2006 Sep;36(9):1336]. Psychological Medicine 36 (2006) 1247-52. http://dx.doi.org/10.1017/S0033291706008105.

[36] J. Prasko, J. Horacek, J. Klaschka, et al., Bright light therapy and/or imipramine for inpatients with recurrent non-seasonal depression. Neuroendocrinology Letters 23 (2002) 109-13.

[37] M. Fritzsche, R. Heller, H. Hill, and H. Kick, Sleep deprivation as a predictor of response to light therapy in major depression. Journal of Affective Disorders 62 (2001) 207-15. http://dx.doi.org/10.1016/S0165-0327(00)00154-3.

[38] A. Baumgartner, H.-P. Volz, A. Campos-Barros, et al., Serum Concentrations of Thyroid Hormones in Patients with Nonseasonal Affective Disorders Treatment with Bright and Dim Light. Society of biological psychiatry 40 (1996) 899-907.

[39] R. Lieverse, E.J.W. Van Someren, M.M.A. Nielen, et al., Bright light treatment in elderly patients with nonseasonal major depressive disorder: A randomized placebo-controlled trial. Archives of $\begin{array}{llll}\text { General } & \text { Psychiatry } & 68 & \text { (2011) }\end{array}$ http://dx.doi.org/10.1001/archgenpsychiatry.2010.183.

[40] N. Goel, M. Terman, J.S. Terman, M.M. Macchi, and J.W. Stewart, Controlled trial of bright light and negative air ions for chronic depression. Psychological Medicine 35 (2005) 945-55. http://dx.doi.org/10.1017/S0033291705005027.

[41] F. Benedetti, C. Colombo, A. Pontiggia, et al., Morning light treatment hastens the antidepressant effect of citalopram: a placebocontrolled trial. Journal of Clinical Psychiatry 64 (2003) 648-53. http://dx.doi.org/10.4088/JCP.v64n0605.

[42] E. Holsboer-Trachsler, U. Hemmeter, M. Hatzinger, et al., Sleep deprivation and bright light as potential augmenters of antidepressant drug treatment: Neurobiological and psychometric assessment of course. Journal of Psychiatric Research 28 (1994) 381-399 available online: http://dx.doi.org/10.1016/0022-3956\%2894\%29900205 .

[43] J.W. Stewart, F.M. Quitkin, M. Terman, and J.S. Terman, Is seasonal affective disorder a variant of atypical depression? Differential response to light therapy. Psychiatry Research 33 (1990) 121-8. http://dx.doi.org/10.1016/0165-1781(90)90065-D.

[44] K. Martiny, M. Lunde, M. Unden, H. Dam, and P. Bech, Adjunctive bright light in non-seasonal major depression: Results from clinician-rated depression scales. Acta Psychiatrica Scandinavica 112 (2005) 117-125. http://dx.doi.org/10.1111/j.16000447.2005.00574.x. 
[45] R.W. Lam, M. Terman, and A. Wirz-Justice, Light therapy for depressive disorders: Indications and efficacy, in Mood disorders: Systematic medication management. 1997, Karger; Switzerland: Basel, Switzerland. 215-234.

[46] A. Wirz-Justice, P. Graw, K. Krauchi, et al., 'Natural' light treatment of seasonal affective disorder. Journal of Affective Disorders 37 (1996) 109-20. http://dx.doi.org/10.1016/0165-0327(95)00081$\underline{\mathrm{X}}$.

[47] K.A. Roecklein, P.M. Wong, M.A. Miller, et al., Melanopsin, photosensitive ganglion cells, and seasonal affective disorder. Neuroscience and Biobehavioral Reviews 37 (2013) 229-239. http://dx.doi.org/10.1016/j.neubiorev.2012.12.009.

[48] T.T. Postolache and D.A. Oren, Circadian phase shifting, alerting, and antidepressant effects of bright light treatment. Clinics in Sports Medicine $24 \quad$ (2005) 381-413, xii. http://dx.doi.org/10.1016/j.csm.2004.12.005.

[49] M.R. Nelsen and D.L. Dunner, Treatment resistance in unipolar depression and other disorders: Diagnostic concerns and treatment possibilities. Psychiatric Clinics of North America 16 (1993) 541 566.

[50] N.S. Philip, L.L. Carpenter, A.R. Tyrka, and L.H. Price, Pharmacologic approaches to treatment resistant depression: A reexamination for the modern era. Expert Opinion on Pharmacother$\begin{array}{llll}\text { apy } & 11 & \text { (2010) } & 709-722 .\end{array}$ http://dx.doi.org/10.1517/14656561003614781.

[51] A. Wirz-Justice and R.H. Van den Hoofdakker, Sleep deprivation in depression: what do we know, where do we go? Biological Psychiatry 46 (1999) 445-53. http://dx.doi.org/10.1016/S00063223(99)00125-0. 